\title{
Proteção por Pré-provisionamento em Redes Ópticas Elásticas
}

\author{
Paulo J. S. Júnior ${ }^{1}$, André C. Drummond ${ }^{2}$ \\ ${ }^{1}$ Instituto Federal de Brasília - Planaltina-DF \\ ${ }^{2}$ Departamento de Ciência da Computação Universidade de Brasília \\ $\{$ paulo.jose@etfbsb.edu.br, andred@unb.br\}
}

\begin{abstract}
With the emergence of EON networks, protection techniques used for WDM networks are reevaluated and research of new techniques gains importance. This work aims to explore the main protection techniques and to compare offline, pre-provisioning, and online, provisioning, adapting the strategies taken from the literature to EON networks. This study explores the realistic view of predicting traffic and adapting routing techniques to this premise. This work presents a heuristic for routing and spectrum allocation for pre-provisioning of DLP, Dedicated Link Protection, and DPP, Dedicated Path Protection. Results show that the use of pre-provisioning techniques in EON obtains good results, compared to the classic provisioning solutions.
\end{abstract}

Resumo. Com o surgimento das redes EON, as técnicas de proteção exploradas para as redes WDM são reavaliadas e a investigação por novas técnicas toma nova importância. Este trabalho objetiva explorar as principais técnicas de proteção e comparar a forma offline, pré-provisionamento, e online, provisionamento, adaptando as estratégias tomadas pela literatura para as redes EON. Este estudo explora a visão realista de se prever o tráfego e adaptar as técnicas de roteamento a essa premissa. São apresentadas heurísticas para roteamento e alocação de espectro para pré-provisionamento de proteção DLP, Proteção Dedicada por Enlace, e DPP, Proteção Dedicada por Caminho. Resultados mostram que o uso de técnicas de pré-provisionamento em EON obtém bons resultados, comparados às soluções clássicas de provisionamento.

\section{Introdução}

Existe um volume bem menor de estudos em proteção para redes EON que paras as redes WDM. Muitas aplicações de proteção em redes WDM ainda estão sendo avaliadas, e seus resultados podem apresentar algumas diferenças com relação às redes EON, devido ao maior número de variáveis e flexibilidade da tecnologia. Existem muitas soluções de provisão de proteção, mas poucos estudos sobre pré-provisionamento. Além disso, nos estudos para redes WDM, ainda faltam avaliações que comparam as principais modalidades de proteção no âmbito do pré-provisionamento. Este trabalho propõe destacar a vantagem do pré-provisionamento de proteção e comparar dois tipos de modalidades de proteção que são priorizadas na fase de tráfego leve e maior excesso de capacidade, para redes EON.

Hoje em dia, a tecnologia WDM, Multiplexação por Divisão de Comprimento de Onda, tem sido predominante nas redes ópticas. Com o constante aumento das demandas e de aplicações heterogêneas, com diversas granularidades, as redes WDM têm trazido 
limitações na eficiência do uso do espectro, ou seja, as aplicações de alta capacidade e as de baixa capacidade não estão aproveitando os recursos das redes WDM de forma eficiente. As redes EON, Elastic Optical Network, vêm surgindo como uma nova solução para prover maior eficiência às redes ópticas. Esta nova tecnologia se destaca pela flexibilidade dos caminhos óticos proverem capacidades diferentes entre si. Muitas técnicas utilizadas nas redes WDM estão sendo adaptadas e recriadas para as redes EON. Nas redes EON, as soluções tendem a ser mais complexas que nas redes WDM devido à sua maior flexibilidade e mais opções de recursos.

As redes EON também apresentam problemas de roteamento, alocação de banda e agregação de tráfego parecidos com as redes WDM, porém a alocação deixa de ser somente de comprimento de onda para ser alocação de espectro e/ou nível de modulação, ou seja, de RSA, Roteamento e alocação de Espectro, e/ou RMLSA, Roteamento, Nível de Modulação e Alocação de Espectro. Neste caso, como a escolha do recurso é mais flexível, os algoritmos de RMLSA tentam encontrar uma rota e um número de slots suficiente para atender a demanda com largura de banda e nível de modulação apropriado dependendo da capacidade e distância a transmitir. O problema RMSLA é um problema NP-difícil [Wang et al. 2011] mais desafiador que o problema de RWA, Roteamento e Alocação de Comprimento de Onda, das redes WDM. Nas redes EON, o problema de restrição de continuidade continua a existir, porém acrescido do problema de contiguidade dos slots. Com isso, o problema da fragmentação ressurge devido à alocação esparsa de slots.

Com a rede EON, o desperdício de banda é consideravelmente menor que o da rede WDM, pois as redes EON permitem atender demandas com caminhos ópticos do tamanho mínimo de até um slot, possibilitando prover recursos de acordo com o tamanho da demanda e mantendo um mínimo de desperdício.

Tendo em mente a mudança de tecnologia das redes ópticas e o aumento das demandas na Internet, a importância das técnicas de sobrevivência torna-se ainda maior. As principais técnicas de sobrevivência das redes EON continuam as mesmas que nas redes WDM. Solucionar uma falha depende de uma sequência de operações que tomam tempo e têm uma grande influência sobre a inatividade da rede. Até se chegar ao restabelecimento de uma conexão que falhou, a rede precisa identificar, localizar, isolar, disponibilizar o backup ou caminho secundário, e decidir se voltará a usar o caminho primário antigo ou criar um novo caminho primário [Mas et al. 2005]. Para este processo ser eficiente, a escolha do tipo de proteção tem muita importância.

Para as redes EON, as técnicas de proteção e restauração continuam prevalecendo e têm suas variações conforme [Shen et al. 2016]. A robustez das redes ópticas é provida através das técnicas de proteção. A proteção é uma solução proativa, antes da falha, onde o caminho secundário é pré-configurado durante a configuração da conexão. A restauração é uma solução reativa, depois da falha, que tenta descobrir o caminho secundário dinamicamente. A proteção tenta uma garantia antes da falha e a restauração não tem garantia por ser no momento da falha. As duas técnicas também dependem da disponibilidade dos recursos no momento de sua implementação, sendo que a proteção reserva recursos antecipadamente e após sua completa implementação a proteção é garantida, enquanto a restauração tenta reservar no momento da falha e sua implementação não é garantida.

A técnica de proteção é a principal solução para prover alta disponibilidade, prin- 
cipal parâmetro para medição da qualidade de serviços em SLA, acordo de níveis de serviços [Bhagavath and Sobol 1992]. A disponibilidade é a probabilidade de um caminho óptico estar disponível em qualquer tempo. A disponibilidade pode ser calculada como

$$
\begin{gathered}
A=\frac{T-T_{\text {off }}}{T} \\
T_{\text {off }}=T_{\text {Detectar }}+T_{\text {Recuperar }}
\end{gathered}
$$

onde $T$ é o tempo total de provisionamento e $T_{\text {off }}$ é o tempo total para o restabelecimento da conexão que falhou. De acordo com esta equação, o $T$ depende da capacidade computacional da rede e é difícil de saber o valor antecipadamente, mas o $T_{\text {off } f}$ pode ser diminuído de acordo com a técnica de proteção adotada [Chen et al. 2014]. As técnicas de proteção têm suas performances avaliadas através das métricas $S T$, tempo de configuração dos caminhos primários e secundários, $P T S$, tempo de comutação das proteções, $P S T$, e $A$, disponibilidade, [Dikbiyik et al. 2012].

Estabelecer proteção pode ser definido como conseguir um caminho secundário para uma conexão origem-destino requisitada. A seleção de um caminho secundário pode ser implementada através de técnicas de provisionamento, reprovisionamento e preprovisionamento, [Dikbiyik et al. 2010]. O provisionamento e o reprovisionamento são tentativas online de proteger, e o pré-provisionamento é uma tentativa offline de proteger futuras demandas. Todas estas três técnicas priorizam melhorar a disponibilidade da rede, ou seja, reduzir o tempo de configuração e o tempo de recuperação. Estas três técnicas dependem da eficiência dos algoritmos de roteamento e alocação de espectro, e são normalmente utilizadas quando da mudança do estado da rede. O estado da rede muda normalmente quando da chegada de novas conexões, término de alguma conexão, falha, reparação e upgrade da rede. Para uma também utilização mais eficiente destas três técnicas, os autores da literatura optaram em fazer o provisionamento sempre quando da chegada de uma nova conexão, o pré-provisionamento ao término de uma conexão, e o reprovisionamento utilizando parâmetros mais complexos que definem quando, como e o que reprovisionar. Todos estes métodos dependem do excesso de capacidade disponível e da taxa de disponibilidade a ser alcançada.

A proteção pode ser dedicada ou compartilhada, total ou parcial, e por caminho, por enlace ou por segmento. A figura 1 apresenta as principais técnicas de proteção.

As proteções dedicadas por enlace e caminho, DLP e DPP, consomem mais recursos que as proteções compartilhadas por enlace e caminho, SLP e SPP, mas são menos complexas de implementar por terem menos restrições. Uma restrição comum para estas duas formas de proteção é que os caminhos primários e backup sejam disjuntos entre si. A proteção compartilhada apresenta restrição de maior complexidade, onde o caminho backup deve pertencer a caminhos primários disjuntos entre si, ou seja, que não façam parte do mesmo grupo de risco. Com isso, o backup pode atender a qualquer falha única ou não simultânea em um dos caminhos primários. A proteção por enlace consome mais recursos que a por caminho, mas tem um tempo de recuperação melhor que a proteção por caminho. A proteção por enlace é mais gulosa e tem uma maior dificuldade de solução que a proteção por caminho. A tabela 1 apresenta um quadro comparativo. 


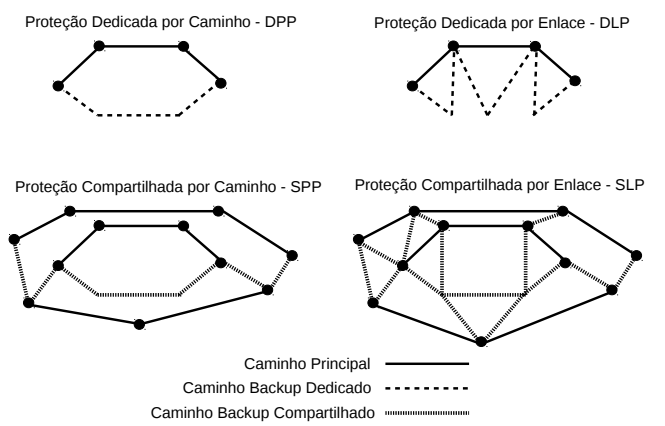

Figura 1. Principais Técnicas de Proteção

Tabela 1.

Consumo de Recurso

$D L P>D P P>S L P>S P P$

Complexidade

$S L P>S P P>D L P>D P P$

Tempo de Recuperação

$D L P<S L P<D P P<S P P$

Este artigo tem como objetivo principal adaptar a técnica de proteção para falha única de fibra óptica com pré-provisionamento [Dikbiyik et al. 2014] das redes WDM para redes EON, destacar a eficiência das redes EON, e justificar a vantagem do pré-provisionamento com relação ao provisionamento no caso das proteções. [Dikbiyik et al. 2014] destaca o pré-provisionamento apenas para melhorar o $S T$ para um contexto onde o tráfego é menos intenso e sob a visão do excesso de capacidade. Nosso estudo observa que o pré-provisionamento pode ser explorado não apenas para melhorar o $S T$, como também para melhorar a disponibilidade e a performance da rede. Um novo algoritmo de pré-provisionamento para redes EON foi desenvolvido.

O artigo está organizado da seguinte forma: na Seção 2 são apresentados trabalhos relacionados; a Seção 3 introduz os algoritmos; na Seção 4 são derivados resultados através de simulações para avaliar a eficiência das soluções apresentadas; e finalmente, na Seção 5 são desenhadas as conclusões.

\section{Trabalhos Relacionados}

Com o aumento das demandas em 100\% a cada dois anos [Saleh and Simmons 2011], alguns estudos vêm destacando a necessidade de mudança ou evolução da tecnologia de transmissão. Em pouco tempo as redes WDM não suportarão as taxas de $400 \mathrm{~GB} / \mathrm{s}$ e $1 \mathrm{~Tb} / \mathrm{s}$ previstas [Gerstel et al. 2012]. As redes EON exploram novas tecnologias de transmissão como a OFDM, Multiplexação por Divisão de Frequência Ortogonal, [Zhang et al. 2013]. A OFDM possibilita transmissões com diferentes modulações e apresenta soluções mais adaptáveis a diferentes demandas. Os equipamentos de transmissão poderão utilizar largura de bandas de acordo com a capacidade necessária das aplicações através da técnica OFDM [Jinno et al. 2010]. Outra mudança importante é a diminuição do slot de frequência de $50 \mathrm{GHz}$ para $12,5 \mathrm{GHz}$, o que facilita o estabelecimento de mais canais de transmissão e de diferentes capacidades. O objetivo desta nova tecnologia é 
possibilitar a utilização de diferentes canais de transmissão com quantidades diferentes de slots para diversas granularidades. [Zhang et al. 2013] apresentam um survey sobre redes EON.

Apesar do melhor aproveitamento da capacidade da fibra, as redes EON trouxeram o problema de fragmentação. As redes WDM trouxeram a solução para fragmentação que ocorria nas tecnologias anteriores devido à técnica TDM, Multiplexação por Divisão de Tempo [Acharya et al. 2004]. No estudo [Rosa et al. 2012] é quantificada a fragmentação nas redes EON. Diferentes políticas de alocação são comparadas e é proposta uma nova política de alocação de espectro com base na comparação de diferentes estratégias de fragmentação. Os autores apresentam funções de avaliação da fragmentação baseada nos estudos de [Wilson et al. 1995].

[Cerutti et al. 2004, Yen and Lee 2007] destacam a solução de agregação de tráfego para redes WDM, e em [Liu et al. 2012] é investigado o problema de agregação de tráfego para redes EON. Este estudo aplica uma técnica eletrônica de ter uma parte de sua capacidade reservada para backup, Shared Spectrum Overlap. Esta técnica permite que o canal adjacente de outro caminho óptico possa ser expandido, invadindo o limite do outro slot sem sobrepor ao espectro. Estes novos transponders são equipamentos adaptados às redes EON que permitem a expansão e contração dos caminhos ópticos.

Estudos anteriores sobre proteção em redes EON são explorados. Em [Sone et al. 2011], uma espécie de proteção squeeze é proposta com consumo de menos banda para proteção dedicada por caminho. Uma sobrevivência por multicaminho é demonstrada em [Ruan and Xiao 2013]. A proteção compartilhada é investigada em [Shao et al. 2012] com heurística de K-shortest Path e alocação First-fit. A proteção dedicada é explorada em [Walkowiak et al. 2014]. Em [Castro et al. 2012] DPP, Proteção Dedicada por Caminho, e SPP, Proteção Compartilhada por Caminho, são comparadas.

O artigo [Dikbiyik et al. 2010] apresenta técnicas de gerenciamento de excesso de capacidade. $\mathrm{O}$ excesso de capacidade, $E C$, é a capacidade não utilizada em uma rede. Neste estudo, são propostas técnicas de gestão de $E C$ para melhorar o desempenho da rede e são apresentadas as principais modalidades de proteção. As proteções são comparadas com relação às variáveis de $S T$, tempo de configuração, $P S T$, tempo de comutação de tipo de proteção e, $A$, disponibilidade. [Dikbiyik et al. 2012] destaca as fórmulas para calcular a disponibilidade das proteções DLP, DPP, SLP e SPP. Todas estas fórmulas têm as indisponibilidades dos caminhos e enlaces principais dependentes da disponibilidade dos caminhos e enlaces de backup, destacando mais ainda as soluções de proteção. O estudo também apresenta solução para melhorar o $S T$ através do pré-provisionamento dos caminhos primário e de backup por $D L P$, e outra solução de reprovisionamento que migra dinamicamente as conexões de proteção de backup. O estudo procura priorizar soluções de alta disponibilidade para quando houver maior excesso de capacidade na rede, e as de menor disponibilidade quando o grau de congestão é alto. Assim, vários esquemas de proteção podem coexistir na rede. A Tabela 2 mostra a performance das modalidades de proteção com relação às variáveis estudadas em [Dikbiyik et al. 2010].

Em [Christodoulopoulos et al. 2011], é provado que o problema de RMLSA é NP-completo e em [Dikbiyik et al. 2010] são apresentadas tabelas que mostram que os resultados de soluções com PLI, Programação Linear Inteira, além de não ser escalável para grande massa de dados, tem resultados muito próximos das heurísticas e com tempo 
Tabela 2.

$S T_{D L P}>S T_{D P P}>S T_{S L P}=S T_{S P P}$

$P S T_{D L P}<P S T_{S L P}<P S T_{D P P}<P S T_{S P P}$

$A_{D L P}>A_{D P P}>A_{S L P}>A_{S P P}$

de implementação cinco vezes maior, sendo praticamente inviável para soluções online.

\section{Heurística de Pré-provisionamento}

\subsection{Pré-provisionamento}

O pré-provisionamento é um método que busca reservar recursos para conexões futuras. A estratégia da reserva deve se basear em um conhecimento prévio do tráfego baseado na técnica de [Dikbiyik et al. 2014] que utiliza uma matriz tráfego da rede USANet. Esta matriz temdados estatísticos dos volumes de dados transportados entre todos os nós da rede. A literatura utiliza a técnica de ordenar o tráfego por pares de nós, atendendo os de maior tráfego prioritariamente. [Dikbiyik et al. 2014] faz apenas o pé-provisionamento DLP em momentos onde o tráfego da rede é menor e onde existe um maior excesso de capacidade. O pré-provisionamento é executado quando da mudança de estado da rede e apenas ao término de qualquer conexão para evitar excesso de processamento e aproveitar a liberação de mais recursos, conforme [Dikbiyik et al. 2014].

\subsection{Heurística}

As heurísticas propostas são para provisionamento de proteção, BProEON, que é online por executar sempre que chega uma requisição de conexão, e para o pré-provisionamento de proteção, BPreEON, que é offline por não atender nenhuma requisição e sim reservar a proteção para futuras chegadas de requisição tanto para DLP como para DPP. Ambos algoritmos recebem como parâmetros, a topologia física, as requisições de conexão com a banda, nós origem e destino, e o tipo ou modo de proteção. Estes algoritmos foram projetados para cenários de tráfego dinâmico e objetivam prover proteção total empregando roteamento e alocação de espectro, RSA, agregação de tráfego, caminhos ópticos com singlehop, salto único, e multihop, vários saltos, considerando a métrica de fragmentação para escolha dos caminhos conforme Equação a seguir.

$$
F_{\text {est }}=1-\frac{c \times \operatorname{Livre}(c)}{\text { TotalLivre }}
$$

, onde c é o número de slots necessários para uma determinada conexão, e Livre(c) é uma função que conta o número de solicitações simultâneas de tamanho c que podem ser satisfeitas. Esta equação é válida no pressuposto de que há sempre um espaço livre no sistema. Para esta Equação, cada classe de solicitação com o número de slots solicitados tem sua própria medida da fragmentação.

No exemplo da literatura [Dikbiyik et al. 2014], o critério de pré-provisionamento prioriza os caminhos com maior número de comprimentos de ondas livres para proteger o máximo possível. Os nossos algoritmos priorizam os caminhos com menor fragmentação. A implementação de rotas singlehop é prioritária e tem como solução secundária as rotas multihop, que resolvem a indisponibilidade de recursos fim-a-fim de um par origemdestino com relação à continuidade e contiguidade de espectro. A agregação de tráfego vai 
acontecer quando uma chamada de um par origem-destino chega na rede e um caminho para este mesmo par ainda esteja alocado pela rede, evitando nova conexão e economia de recurso.

Os nossos algoritmos de pré-provisonamento são uma adaptação de [Dikbiyik et al. 2014] para redes EON. No pré-provisionamento em [Dikbiyik et al. 2014], a opção foi pela escolha dos caminhos de menor distância combinado com os caminhos de menor número de comprimentos de ondas livres. Em [Dikbiyik et al. 2014], é reservado um caminho secundário para cada comprimento de onda de cada par, e com isto, tem-se a garantia de backup para qualquer próxima demanda ou granularidade que seja menor ou igual ao comprimento de onda. No caso do nosso algoritmo de pré-provisionamento, BPreEON, a capacidade a ser reservada sempre será igual ou maior que o tamanho da classe de serviço com maior granularidade, garantindo assim atender a qualquer demanda. Ambos algoritmos utilizam o algoritmo $k$-shortest paths do [Yen 1971]. Nossos algoritmos utilizam a técnica de menores caminhos junto com a escolha do caminhos menos fragmentados para melhorar o desempenho. Além de se adaptarem às redes EON, nossas heurísticas trazem como novidade a escolha dos caminhos secundários reservando a capacidade da granularidade máxima e a utilização do custo de fragmentação para escolha dos melhores caminhos.

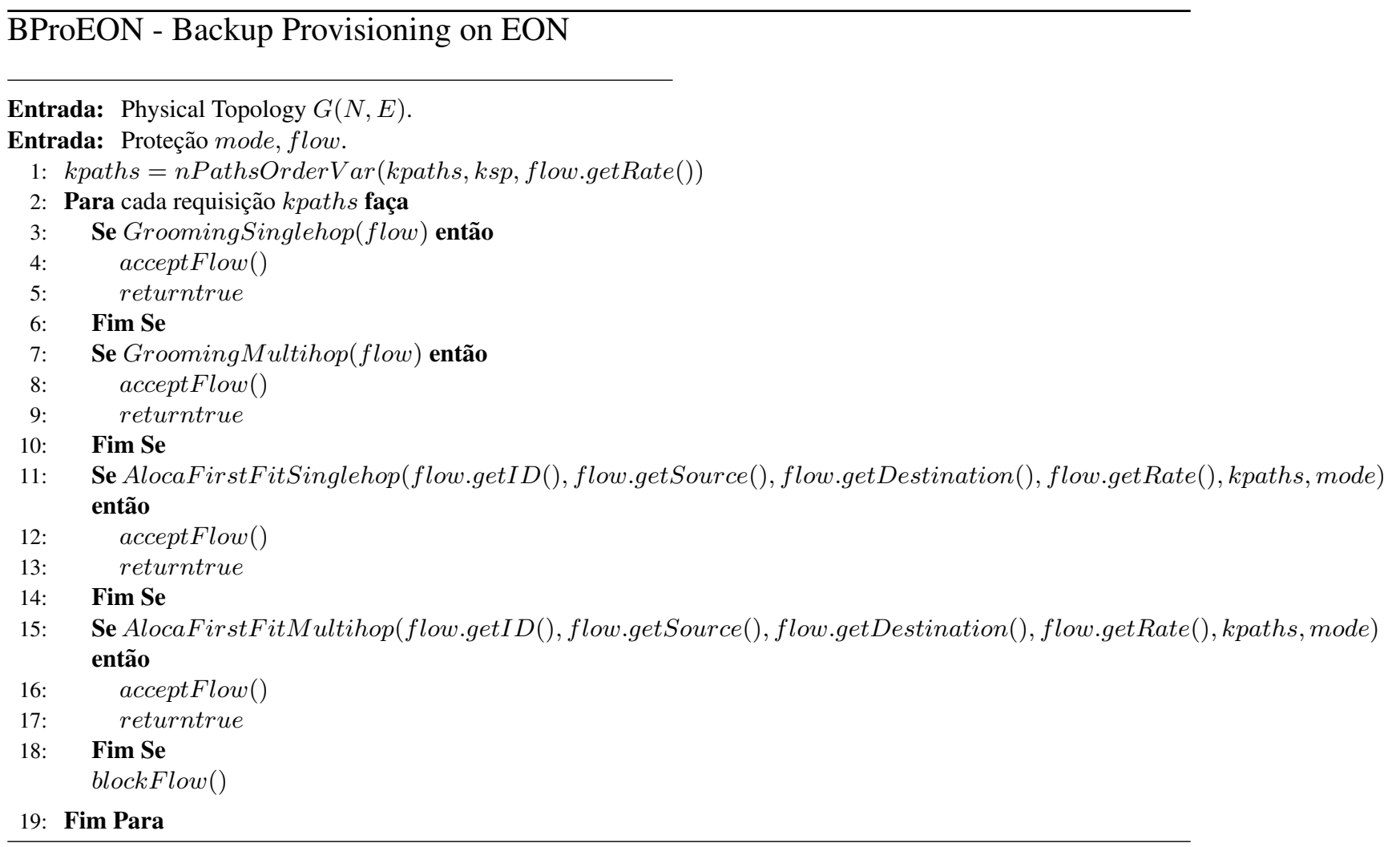

O algoritmo de provisionamento BProEON mostra uma sequência de métodos: agregação em caminho singlehop, agregação em caminho multihop, alocação por caminho singlehop e finalmente alocaçao por multihop. A forma de alocação é o first-fit para todos os algoritmos. Os parâmetros de entrada são a topologia física e a conexão representada pelo seu fluxo que traz os nós pares e sua banda.

O algoritmo BPreEON é executado antes de qualquer requisição de conexão che- 


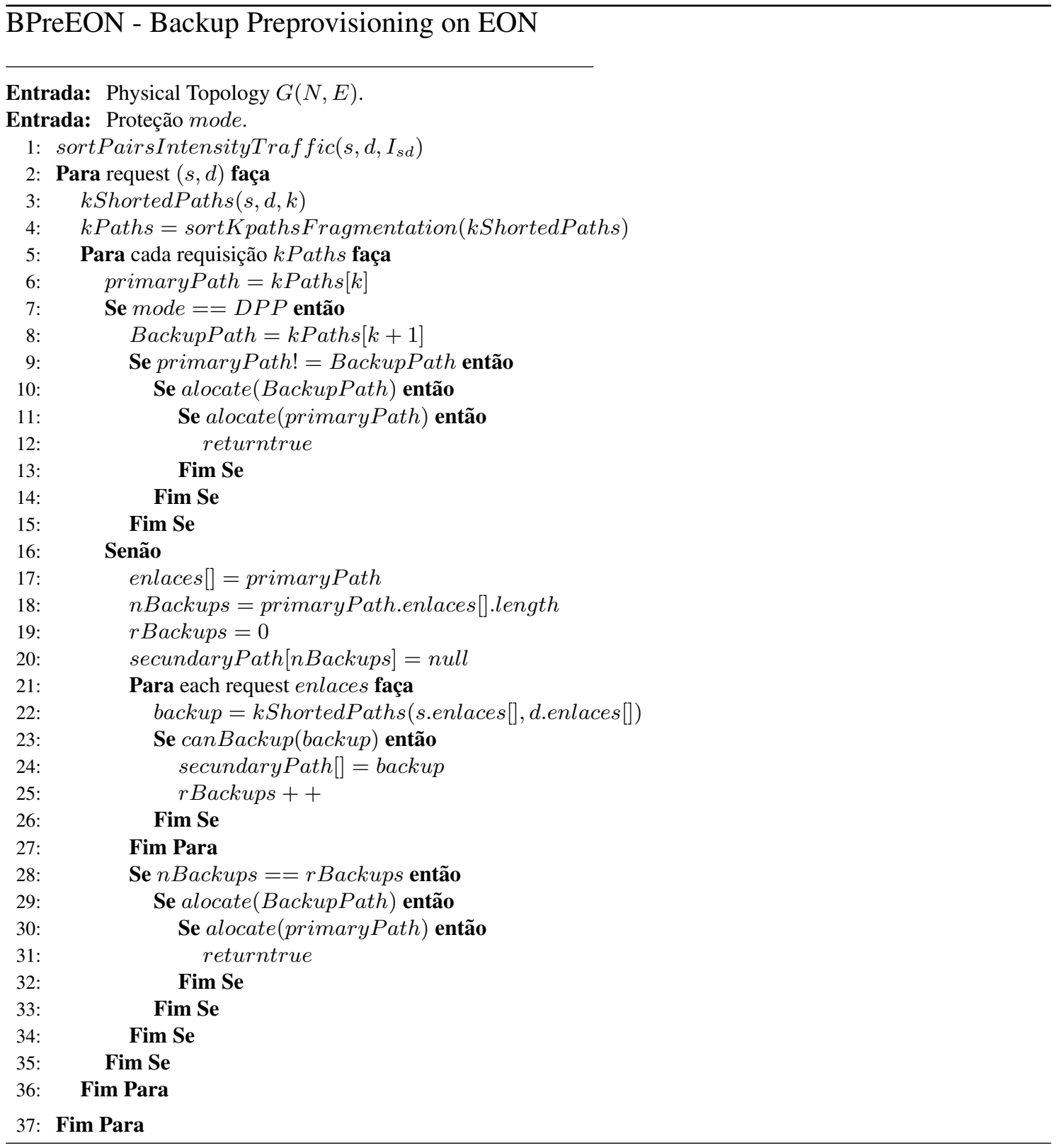

gar à rede, ou sempre que alguma conexão for liberada da rede, ou seja, assim que um fluxo chegar ao seu destino. O BProEON é o programa principal e o BPreEON é o BProEON acrescido do roteamento estático para pré-provisionamento da proteção.

O BPreEON recebe como entrada a topologia $G(N, E)$, onde $N$ é o conjunto de nós e $E$ o conjunto de enlaces e o tipo de proteção, mode. O BProEON como parte do BPreEON tem as funções básicas de RSA para singlehop e multihop junto com a agregação de tráfego. O BProEON tenta primeiro agregar, pois à medida que vão chegando as requisições de conexão, o algoritmo verifica a existência de caminhos ópticos disponíveis para mesma origem e destino. A função GroomingSinglehop(flow), de agregação por salto único ou único caminho óptico, deve encontrar todos os conjuntos de caminhos ópticos, dado o conjunto de todos os caminhos ópticos existentes, para um determinado par origem-destino. Este problema pode ser mapeado no problema clássico de empacotamento de conjuntos, que é NP-Completo. A função 
GroomingMultihop(flow), de agregação de saltos múltiplos ou mais de um caminho óptico, deve encontrar todos os conjuntos de caminhos ópticos, dado o conjunto de todos os caminhos ópticos existentes, que tenha ou mesma origem ou mesmo destino, e que entre eles uma origem de um seja igual ao destino do outro ou vice-versa pra identificálos como conectados entre si. A função AlocaFirstFitSinglehop(...), avalia todos os caminhos de kpaths, e tenta encontrar um caminho com o número de slots contínuos e contíguos, ou seja, tenta provisionar uma proteção utilizando apenas um caminho óptico. A função AlocaFirstFitMultihop(...) tenta criar multicaminhos da mesma forma que a função GroomingMultihop(flow).

O BPreEON acrescenta ao BProEON o roteamento estático da proteção. Este roteamento é feito sempre que uma requisição de conexão chega ou sai da rede, ou seja, o roteamento, apesar de ser feito de forma offline e estático, se adapta dinamicamente ao tráfego. O BPreEON recebe como entrada a topologia $G(N, E)$, onde $N$ é o conjunto de nós e $E$ o conjunto de enlaces. Dado um conjunto de pares origem-destino, $\rho=\{$ $\left.s, d, I_{s d}\right\}$ onde $s$ é o nó origem e $d$ é o nó destino, $I_{s d}$ é a intensidade de tráfego medida entre os pares. Na linha 1 , o método sortPairsIntensityTraffic $\left(s, d, I_{s d}\right)$ gera uma lista de pares s-d ordenada decrescentemente por intensidade de tráfego. Esta lista servirá para um novo ordenamento na linha 3 que gerará K menores caminhos para cada par s-d ordenados por distância e na linha 4 novamente por fragmentação. A variável $k$ pode ser crescente de acordo com a necessidade. Depois, para cada um dos $\mathrm{K}$ caminhos, o algoritmo define o caminho primário e um outro caminho de backup disjuntos entre si. Caso o tipo de proteção seja DPP, linha 7 a 15, teremos apenas um caminho primário e um caminho backup, caso o modo seja DLP, linha 16 a 35, será criado um caminho primários e um caminho backup para cada enlace deste caminho primário escolhido. No caso do pré-provisionamento, o caminho primário somente será reservado caso possibilite a criação do backup, ou seja, o objetivo é somente reservar caminhos protegidos. O préprovisionamento reserva um conjunto de slots que atenda à maior granularidade de forma a garantir qualquer conexão futura para os pares de nós protegidos.

\section{Resultados Numéricos}

Tomando como base comparativa o artigo de [Dikbiyik et al. 2014], as proteções que têm melhor $S T$, a DLP e DPP, são avaliadas e comparadas entre o provisionamento e o pré-provisionamento. Apesar da adaptação, o nosso algoritmo optou em não comparar os resultados devido às diferentes características das redes WDM e EON. Para avaliar o desempenho dos algoritmos propostos foram realizadas simulações utilizando o simulador de redes ópticas ONS [Costa L. e Sousa L. e Oliveira F. e Silva K. e Souza P. e Drummond 2016]. Foram implementados os algoritmos de provisionamento e de pré-provisionamento de proteção.

Cada simulação foi realizada 5 vezes utilizando o método de replicações independentes. Para os resultados apresentados foram calculados intervalos de confiança com 95\% de confiabilidade. Em cada simulação foram geradas 10.000 requisições de conexão para diferentes níveis de carga na rede. A carga, medida em Erlang, é definida como a taxa média de chegada $\times$ a duração da chamada $\times$ a banda passante da chamada normalizada pela capacidade do enlace. As principais métricas utilizadas foram a taxa média de bloqueio de banda (MBBR) e o percentual de proteção obtida por chamada aceita na rede. Para cada evento de chegada simulado, os algoritmos foram executados 
levando em consideração a origem, destino, demanda de banda passante e com proteção total DLP. Caso o algoritmo encontre uma solução, os recursos são alocados na rede e a chamada aceita, caso contrário, a chamada é bloqueada. A topologia consideradas nas simulações foi a USANet com 24 nós e 43 enlaces bidirecionais, Figura 2. As granularidades foram divididas em $200 \mathrm{Mb}, 10 \mathrm{~Gb}$ e $20 \mathrm{~Gb}$ e distribuídas em demandas uniformes. A modulação empregada é a QPSK, com 128 slots, 2 slots de banda de guarda e tamanho do slot com $12500 \mathrm{Gbit} / \mathrm{sec}$. As cargas testadas são para tráfego baixo e intenso, tendo como parâmetro principal o pré-provisionamento.

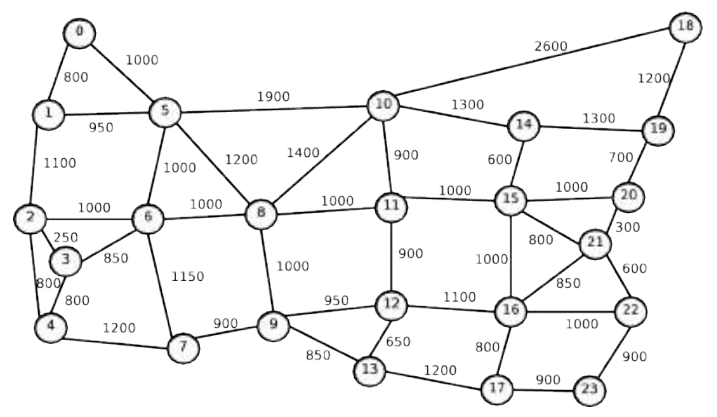

Figura 2. Topologia USANet

Na Figura 3 e na Figura 4, estamos comparando a eficiência entre as redes WDM representada pelo algoritmo BProEON(RWA), e EON, com o algoritmo BProEON(RSA). Observa-se que em altas cargas e nas redes EON, o resultado do BProEON(RSA) é pior que o do BProEON(RWA) devido ao maior dano causado pela fragmentação. Outro resultado a destacar é que as redes WDM têm uma capacidade maior de agregação que as redes EON. Em redes WDM, o RWA tem um resultado bem pior em baixas cargas, mas em altas cargas o resultado é melhor, constatando o dano da fragmentação para as redes EON, principalmente em momento de congestão do tráfego. O objetivo desta comparação é destacar as vantagens e desvantagens entre as tecnologias WDM e EON. Os dois algoritmos também fazem agregação e provisão de um salto e multi-saltos. A topologia física da rede USANnet WDM é de 32 comprimentos de ondas com $50 \mathrm{GHz}$ de espectro e para a rede EON é de modulação BPSK, com 128 slots, 2 slots de banda de guarda e tamanho do slot com $12,5 \mathrm{GHz}$ de espectro.

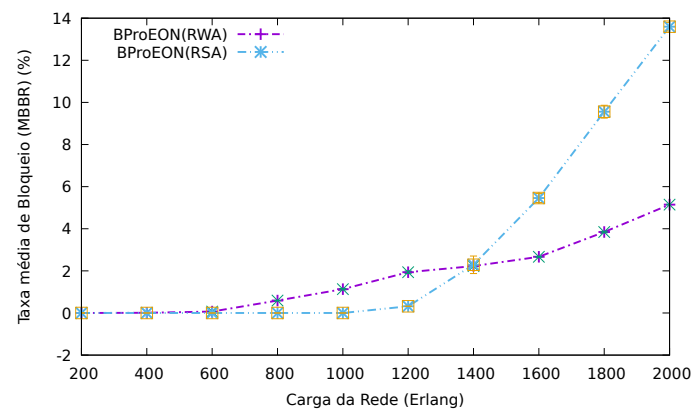

Figura 3. MBBR entre Provisionamento RWA(WDM) e RSA(EON).

Como o objetivo da pesquisa é também melhorar a disponibilidade e o tempo de configuração das proteções, a capacidade de proteção é o alvo principal dos algoritmos propostos. A Figura 5 destaca a eficiência da proteção do pré-provisionamento com relação ao provisionamento. O pré-provisionamento DLP protege em média 4 vezes mais 


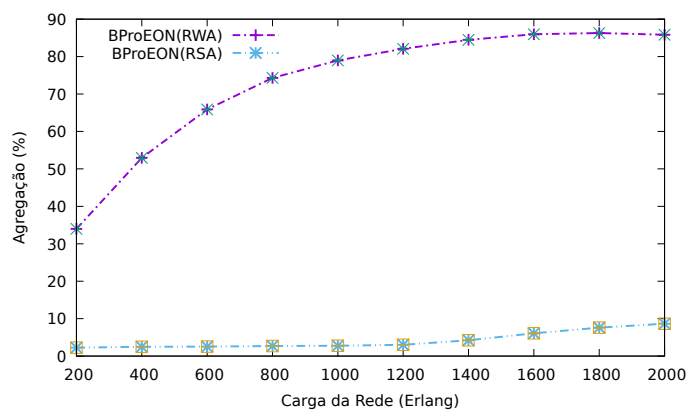

Figura 4. Agregação entre Provisionamento RWA(WDM) e RSA(EON).

que o provisionamento DLP, e o pré-provisionamento DPP protege de três e duas vezes mais que o provisionamento DPP. A proteção dos pré-provisionamentos é bem superior à dos provisionamentos.

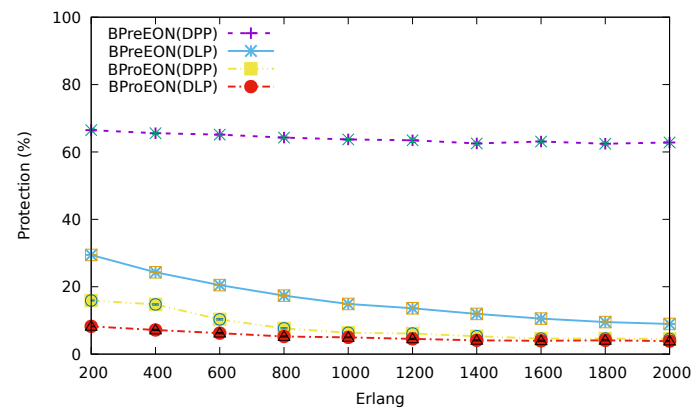

Figura 5. Proteção para Provisionamento e Pré-provisionamento.

Na Figura 5, o pré-provisionamento tem um destaque maior que o provisionamento, principalmente pela eficiência na proteção dos caminhos. A técnica de provisão de tráfego com roteamento estático destaca a quantidade de caminhos protegidos pelo pré-provisionamento sendo bem maior que a quantidade de caminhos protegidos pelo provisionamento. Em todas as técnicas, as proteções vão diminuindo à medida que os recursos vão ficando mais escassos, ou seja, nas altas cargas de tráfego. Outra característica observada é a ineficiência da técnica DLP com relação a DPP devido à sua característica gulosa de reservar backups. Apesar da técnica DLP proteger menos caminhos que a DPP, o número de caminhos de backups necessários para proteger o caminho primário é maior que a técnica DPP. Assim, o rendimento do MBBR para a proteção DPP é superior à DLP.

A Figura 6 mostra a eficiência das proteções por caminho, DPP, sobre as proteções por enlace, DLP, com relação à MBBR. Isto ocorre devido à característica gulosa da proteção por enlace. A técnica DLP propicia um maior consumo de recursos que a DPP. A proteção total e a proteção por enlace consomem muitos recursos da rede, propiciando uma rápida escassez de recursos. Entre todas as técnicas testadas, o pré-provisionamento DPP é o mais eficiente. O pré-provisionamento tem como principal solução fazer o rotamento antecipado e offline de acordo com as características das intensidades de tráfegos de cada par origem-destino. Entre os mesmos tipos de proteção, o pré-provisionamento tem um rendimento melhor que o provisionamento para todas as técnicas.

Na Figura 7, o pré-provisionamento apresenta uma maior agregação de tráfego que 


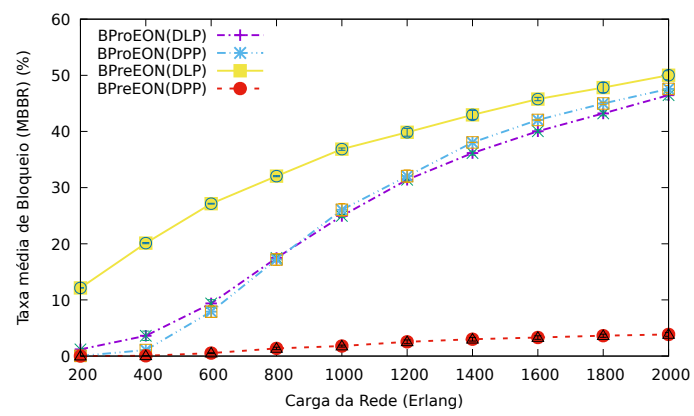

Figura 6. MBBR para Provisionamento e Pré-provisionamento.

o provisionamento, o que é esperado devido à reserva de caminhos com a maior granularidade e de forma prévia às demandas. O pré-provisionamento cria o problema de retirar recursos da rede antes das chegadas das demandas, mas mitiga este desperdício através do aumento da capacidade de agregação. A agregação vai diminuindo à medida que a taxa de bloqueio aumenta e os recursos vão ficando mais escassos devido ao aumento do tráfego.

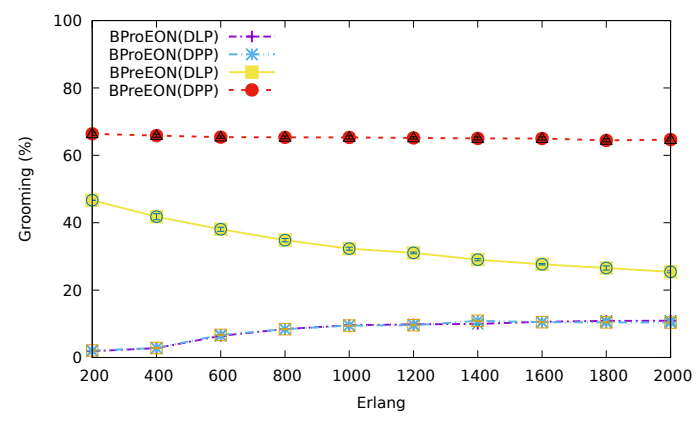

Figura 7. Agregação para Provisionamento e Pré-provisionamento.

\section{Conclusão}

Os autores deste artigo chegaram à conclusão que uma previsão do tráfego sempre ajudará no planejamento para a alocação antecipada de recursos, e principalmente se adaptada às características realistas de tráfego da rede em teste. Algumas vantagens e desvantagens foram destacadas.

O pré-provisionamento tem uma grande vantagem com relação ao provisionamento que é o ajuste de suas reservas com base na previsão do tráfego. O provisionamento tenta a melhor solução apenas no momento da chegada da requisição de conexão. O pré-provisionamento DLP tem um melhor tempo $S T$, mas deve ser utilizado apenas em baixas cargas e quando o excesso de capacidade é alto. O pré-provisionamento DPP protege bem mais que o DLP. A fragmentação é muito danosa em altas cargas e alocar e reservar capacidade com granularidade bem superior, isto é, da mesma forma que nas redes WDM, pode mitigar este problema.

O algoritmo deste estudo confirma a eficiência do pré-provisionamento que é destacado no artigo [Dikbiyik et al. 2014] e abre espaço para melhorar outras variáveis que não o tempo de configuração da proteção. O pré-provisionamento aumenta a capacidade de proteção e consequentemente a disponibilidade da rede. A adaptação do nosso 
algoritmo com a reserva da maior granularidade e a escolha dos caminhos de melhor fragmentação é a chave para a eficiência do algoritmo. O maior desafio para os próximos trabalhos deverá ser testar técnicas de reprovisionamento.

\section{Referências}

Acharya, S., Gupta, B., Risbood, P., and Srivastava, A. (2004). Mobipack: optimal hitless sonet defragmentation in near-optimal cost. In IEEE INFOCOM 2004, volume 3, pages 1819-1829 vol.3.

Bhagavath, V. K. and Sobol, H. (1992). Reliability evaluation and protection schemes for dense wdm network architectures. In Communications, 1992. ICC '92, Conference record, SUPERCOMM/ICC '92, Discovering a New World of Communications., IEEE International Conference on, pages 174-178 vol.1.

Castro, A., Velasco, L., Ruiz, M., and Comellas, J. (2012). Single-path provisioning with multi-path recovery in flexgrid optical networks. In Ultra Modern Telecommunications and Control Systems and Workshops (ICUMT), 2012 4th International Congress on, pages $745-751$.

Cerutti, I., Fumagalli, A., and Sheth, S. (2004). Performance versus cost analysis of wdm networks with dynamic traffic grooming capabilities. In Computer Communications and Networks, 2004. ICCCN 2004. Proceedings. 13th International Conference on, pages $425-430$.

Chen, X., Ji, F., and Zhu, Z. (2014). Service availability oriented p-cycle protection design in elastic optical networks. IEEE/OSA Journal of Optical Communications and Networking, 6(10):901-910.

Christodoulopoulos, K., Tomkos, I., and Varvarigos, E. A. (2011). Elastic bandwidth allocation in flexible ofdm-based optical networks. Journal of Lightwave Technology, 29(9):1354-1366.

Costa L. e Sousa L. e Oliveira F. e Silva K. e Souza P. e Drummond, A. (2016). Ons: Simulador de eventos discretos para redes Ópticas wdm/eon. In: Salão de Ferramentas, 2016, Salvador. XXXIV Simpósio Brasileiro de Redes de Computadores e Sistemas Distribuídos, 2016.

Dikbiyik, F., Sahasrabuddhe, L., Tornatore, M., and Mukherjee, B. (2010). Exploiting excess capacity for improved robustness in optical WDM backbone mesh networks. Optical Fiber Communication (OFC), collocated National Fiber Optic Engineers Conference, 2010 Conference on (OFC/NFOEC), pages 1-3.

Dikbiyik, F., Sahasrabuddhe, L., Tornatore, M., and Mukherjee, B. (2012). Exploiting excess capacity to improve robustness of wdm mesh networks. IEEE/ACM Transactions on Networking, 20(1):114-124.

Dikbiyik, F., Tornatore, M., and Mukherjee, B. (2014). Exploiting excess capacity for survivable traffic grooming in optical backbone networks. IEEE/OSA Journal of Optical Communications and Networking, 6(2):127-137.

Gerstel, O., Jinno, M., Lord, A., and Yoo, S. J. B. (2012). Elastic optical networking: a new dawn for the optical layer? IEEE Communications Magazine, 50(2):s12-s20. 
Jinno, M., Kozicki, B., Takara, H., Watanabe, A., Sone, Y., Tanaka, T., and Hirano, A. (2010). Distance-adaptive spectrum resource allocation in spectrum-sliced elastic optical path network. IEEE Communications Magazine, 48(8):138-145.

Liu, M., Tornatore, M., and Mukherjee, B. (2012). Survivable traffic grooming in elastic optical networks - Shared path protection. IEEE International Conference on Communications, pages 6230-6234.

Mas, C., Nguyen, H. X., and Thiran, P. (2005). Failure Location in WDM Networks, pages 379-399. Springer US, Boston, MA.

Rosa, A., Cavdar, C., Carvalho, S., Costa, J., and Wosinska, L. (2012). Spectrum allocation policy modeling for elastic optical networks. High Capacity Optical Networks and Emerging/Enabling Technologies, pages 242-246.

Ruan, L. and Xiao, N. (2013). Survivable multipath routing and spectrum allocation in ofdm-based flexible optical networks. IEEE/OSA Journal of Optical Communications and Networking, 5(3):172-182.

Saleh, A. A. M. and Simmons, J. M. (2011). Technology and architecture to enable the explosive growth of the internet. IEEE Communications Magazine, 49(1):126-132.

Shao, X., Yeo, Y. K., Xu, Z., Cheng, X., and Zhou, L. (2012). Shared-path protection in ofdm-based optical networks with elastic bandwidth allocation. In Optical Fiber Communication Conference and Exposition (OFC/NFOEC), 2012 and the National Fiber Optic Engineers Conference, pages 1-3.

Shen, G., Guo, H., and Bose, S. K. (2016). Survivable elastic optical networks: survey and perspective (invited). Photonic Network Communications, 31(1):71-87.

Sone, Y., Watanabe, a., Imajuku, W., Tsukishima, Y., Kozicki, B., Takara, H., and Jinno, M. (2011). Bandwidth Squeezed Restoration in Spectrum-Sliced Elastic Optical Path Networks (SLICE). IEEE/OSA Journal of Optical Communications and Networking, 3(3):223-233.

Walkowiak, K., Klinkowski, M., Rabiega, B., and Goścień, R. (2014). Routing and spectrum allocation algorithms for elastic optical networks with dedicated path protection. Optical Switching and Networking, 13:63-75.

Wang, Y., Cao, X., and Pan, Y. (2011). A study of the routing and spectrum allocation in spectrum-sliced elastic optical path networks. pages 1503-1511.

Wilson, P. R., Johnstone, M. S., Neely, M., and Boles, D. (1995). Dynamic storage allocation: A survey and critical review. pages 1-116. Springer-Verlag.

Yen, H.-H. and Lee, S. S. W. (2007). QoS aware traffic grooming and integrated routing on IP over WDM networks. Photonic Network Communications, 14(1):1-10.

Yen, J. Y. (1971). Finding the k shortest loopless paths in a network. Management Science, 17(11):712-716.

Zhang, G., De Leenheer, M., Morea, A., and Mukherjee, B. (2013). A survey on ofdm-based elastic core optical networking. Communications Surveys Tutorials, IEEE, 15(1):65-87. 